\title{
Isolated development of inner (wall) caries like lesions in a bacterial-based in vitro model
}

\author{
K. Diercke • A. Lussi • T. Kersten • R. Seemann
}

Received: 24 September 2008 / Accepted: 12 January 2009 /Published online: 12 February 2009

(C) Springer-Verlag 2009

\begin{abstract}
The study conducted in a bacterial-based in vitro caries model aimed to determine whether typical inner secondary caries lesions can be detected at cavity walls of restorations with selected gap widths when the development of outer lesions is inhibited. Sixty bovine tooth specimens were randomly assigned to the following groups: test group 50 (TG50; gap, $50 \mu \mathrm{m}$ ), test group 100 (TG100; gap, $100 \mu \mathrm{m}$ ), test group 250 (TG250; gap, $250 \mu \mathrm{m}$ ) and a control group (CG; gap, $250 \mu \mathrm{m}$ ). The outer tooth surface of the test group specimens was covered with an acidresistant varnish to inhibit the development of an outer caries lesion. After incubation in the caries model, the area of demineralization at the cavity wall was determined by confocal laser scanning microscopy. All test group specimens demonstrated only wall lesions. The CG specimens developed outer and wall lesions. The TG250 specimens showed significantly less wall lesion area compared to the CG $(p<0.05)$. In the test groups, a statistically significant increase $(p<0.05)$ in lesion area could be detected in enamel between TG50 and TG250 and in dentine between TG50 and TG100. In conclusion, the inner wall lesions of secondary caries can develop without the presence of outer
\end{abstract}

K. Diercke $\cdot$ T. Kersten $\cdot$ R. Seemann

Department of Operative and Preventive Dentistry,

Dental School, Charité,

Berlin, Germany

A. Lussi $\cdot$ R. Seemann $(\bowtie)$

Department of Preventive, Operative and Pediatric Dentistry,

University of Bern,

3010 Bern, Switzerland

e-mail: rainer.seemann@zmk.unibe.ch

K. Diercke

Department of Orthodontics, University of Jena,

Jena, Germany lesions and therefore can be regarded as an entity on their own. The extent of independently developed wall lesions increased with gap width in the present setting.

Keywords Secondary caries · Wall lesion · Inner lesion . Gap size $\cdot$ Artificial mouth

\section{Introduction}

A secondary caries lesion has been defined as a positively diagnosed carious lesion which occurs at the margin of an existing restoration [1]. Histopathologically, secondary caries is described to consist of an outer and an inner lesion or wall lesion $[2,3]$. According to Hals' theory, the outer lesion is caused by the primary caries attack on the tooth surface and appears histologically as a primary caries lesion. The inner lesion, however, is the consequence of micro-leakage [3]. Thus, the inner lesion is the result of bacteria, fluids and hydrogen ions diffusing into the gap between restoration and cavity wall. Wall lesions were also observed in later examinations [4-10].

A contrary theory on the aetiology of secondary lesion denies secondary lesions occurring because of gaps. It assumes that secondary lesions are similar to primary lesions and just differ by their appearance next to restorations. This theory is explained by the direction of the enamel rods in relation to the tooth restoration interface determining the presence of a wall lesion and its extent. [11, 12]. The existence of a wall lesion per se has been considered doubtful [12]. Based on this theory, wall lesion should not develop at gaps with different widths when the development of the outer lesion is inhibited.

Based on the available English and German literature, up to now, the development of wall lesions and the relation 
between gap size and lesion depth has only been studied by considering the inner and outer lesion together. In 1968, Jørgensen and Wakumoto [13] described a close correlation between low secondary caries risk and tight gap sizes. They observed caries at the cavity wall only when the gap exceeds 35-50 $\mu \mathrm{m}$. In the year 1991, a study by Derand et al. [14] followed, which demonstrated higher degrees of demineralization at wider gaps in vitro. Hodges et al. confirmed these results 1995 in vivo. However, an examination by Söderholm et al. [15] contrasted this. The authors mentioned a poor correlation between gap widths and secondary lesion on extracted teeth. Although gaps were very narrow, they observed deep lesions especially in dentine. García-Godoy et al. [5] also measured gap sizes less than $2 \mu \mathrm{m}$ next to secondary carious lesions. A recently published study by Totiam et al. [16] used a microbialbased in vitro caries model with adjustable gap widths and found that the width of the gab between the tooth and the restoration affects the development of secondary caries. However, also in this study, primary lesions and wall lesions were not considered independently. If the existence of inner lesions depends on the development of outer lesions, this would have strong impact on the clinical diagnosis, treatment and prevention of secondary caries.

The present study conducted in a bacterial-based in vitro caries model aimed to determine whether typical inner lesions can be detected at simulated cavity walls with selected gap widths when the development of outer lesions is inhibited.

\section{Materials and methods}

\section{Experimental design}

Fifteen anterior teeth were extracted from six bovine mandibles. Four specimens were derived from each tooth.
The specimens were randomly assigned to one of the following four groups:

Test group 50 (gap width of $50 \mu \mathrm{m}$ ),

Test group 100 (gap width of $100 \mu \mathrm{m}$ ),

Test group 250 (gap width of $250 \mu \mathrm{m}$ ) and

Control group (gap width of $250 \mu \mathrm{m}$ ).

Thus, each group held 15 specimens. The development of an outer lesion was inhibited in the test groups by covering the enamel surface with an acid-resistant varnish (Nivea Flex, NIVEA Beauté Beiersdorf AG, Hamburg, Germany; Fig. 1).

Preparation of specimens

After removal of the roots, the crowns were embedded in a two-component cold embedding resin based on methyl methacrylate (Technovit 4004, Heraeus Kulzer GmbH \& Co., Wehrheim, Germany). Using a diamond saw microtome (Leitz 1600, Ernst Zeitz, Wetzlar, Germany), each tooth was cut in four $3 \times 3 \times 5-\mathrm{mm}$ blocks. The specimens were stored in tap water.

For the production of defined microspaces, a vice-like appliance was constructed at the Medical Research Department of the Charite based on the construction described by Totiam et al. [16]. Our vice-like appliance was made of polyoxymethylene and consists of a static part and a movable part. On the static part, the tooth specimens were mounted with composite (Z100, 3M ESPE, Seefeld, Germany), whilst the movable part simulated the restoration wall. Between both parts, the desired gap width could be adjusted by bringing up the movable part to the static part (Fig. 1). Before the adjustment, all test group specimens' surfaces were covered with a double layer of acid-resistant nail varnish. The correct application of the varnish was checked using a stereo microscope (Stemi 2000, Carl Zeiss, Jena, Germany) with $4 \times$ magnification. For the adjustment
Fig. 1 Drawing of test group vice-like appliance with specimen and varnish on the surface (left side) and a detailed drawing of the area of demineralization measurement in enamel and dentine (right side)

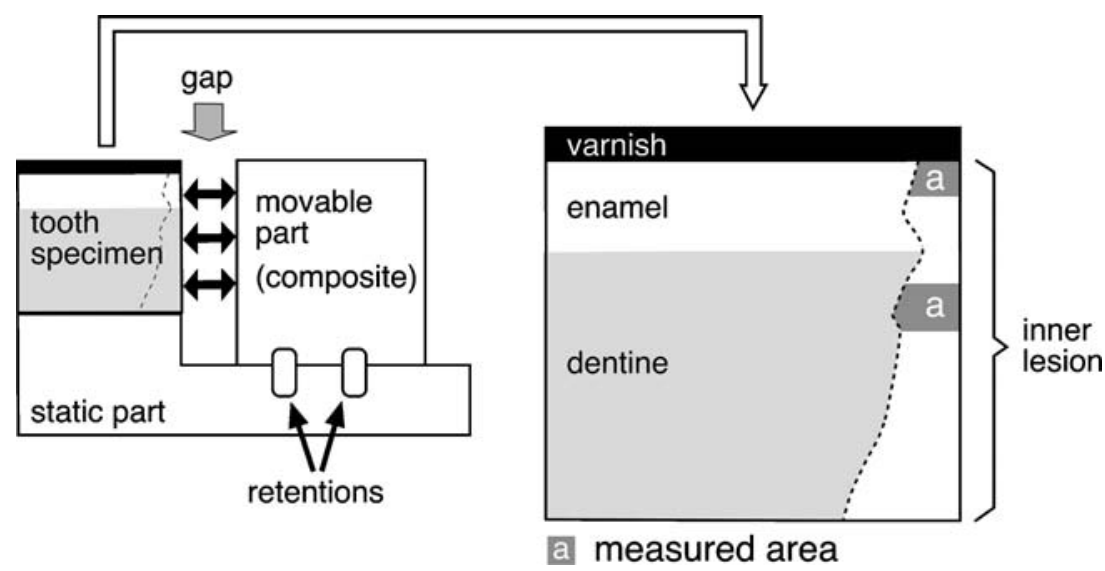


of gaps, metal bands with a thickness of 50, 100 and $250 \mu \mathrm{m}$, respectively, were used. The metal bands were brought between the static part and the movable part and both parts were closed as tight as possible, leaving the metal band between them. By application of composite into lateral retention grooves, the desired gap width was locked and the metal bands were removed. The correct gap width was checked with a calibrated light microscope (Laborlux 12, Ernst Zeitz). Prior to entry in the microbial-based in vitro caries model, the specimens were sterilised with ethylene dioxide as described elsewhere [17].

\section{Microbial-based in vitro caries model}

A detailed description of the microbial-based in vitro caries model has recently been published [17, 18]. Briefly, the initially sterilised specimens were inoculated with Streptococcus mutans (ATCC 25175) for biofilm development and incubated in this caries model for 21 days. The developing plaque-like biofilm received a continuously repeating treatment regime: "trypticase soy broth" (TSB; Becton Dickinson, Sparks, USA) for bacterial growth every $15 \mathrm{~min}$ for $2 \mathrm{~min}$, "artificial saliva" (1 1 of distilled water contained $1.2 \mathrm{~g} \mathrm{KCl} ; 0.84 \mathrm{~g} \mathrm{NaCl} ; 0.26 \mathrm{~g} \mathrm{KH}_{2} \mathrm{PO}_{4} ; 0.15 \mathrm{~g} \mathrm{CaCl}_{2}$; $\mathrm{pH}=6.8$ [19] for remineralisation every $15 \mathrm{~min}$ for $2 \mathrm{~min}$ and " $10 \%$ sucrose solution" as bacterial substrate once every hour instead of TSB. S. mutans bacteria were applied on the first day and the seventh day by dripping $30 \mathrm{ml}$ onto the specimens (flow rate, $0.4 \mathrm{ml} / \mathrm{min}$ ), resulting in approximately $1 \mathrm{ml} /$ specimen.

\section{Inner (wall) lesion evaluation}

The specimens were dismounted from the vice-like appliance and the fixation composite was removed with silicon carbide paper (220 grit). The lateral surfaces (perpendicular to the gap) of the specimens were polished with silicon carbide paper (4000 grit) and were examined with confocal laser scanning microscopy ("Zeiss LSM 510", Carl Zeiss; Fig. 1) [17]. An argon laser (wavelength, $488 \mathrm{~nm}$; intensity, $46 \%$ ), a $2.5 \times$ Fluar objective with a numerical aperture of 0.075 and the following beam path configuration was used: channel 1, long pass (LP650), $386 \mu \mathrm{m}$ pinhole; channel 2, band pass (BP 505-530), 386- $\mathrm{mm}$ pinhole and channel 3, band pass (BP 560-615), 374- $\mathrm{m}$ pinhole. HFT 488 was taken as "main dichroic beam splitter" and NFT 545 as "secondary dichroic beam splitter". There was no need to stain the specimens [17]. The area of the inner lesion (in $\mu \mathrm{m}^{2}$ ) was determined with the help of a special software (Zeiss LSM 510 Image Browser rel. 4.0, Carl Zeiss). Because different specimens showed individual thicknesses of enamel and dentine layers, an area of interest was defined to allow a standardised comparison between groups. Enamel inner lesions were examined in a distance of $0-300 \mu \mathrm{m}$ from the tooth surface and dentine inner lesions from $1250-1550 \mu \mathrm{m}$ along the tooth restoration interface (Fig. 1). One examiner, who was blinded to the specimen group, conducted all measurements.

\section{Statistics}

The Kolmogorov-Smirnov test was used to test for normal distribution of the data. The paired $t$ test with a sequential "Bonferroni correction" by Holm [20] was used to compare the three test groups and the test group 250 with the control group. Values of $P$ lower than 0.05 were considered as significant in all comparisons.

\section{Results}

After 21 days of incubation in the microbial-based in vitro caries model, all specimens were covered by a whitish and yellowish plaque-like biofilm. The waste container and the bottles of media showed no signs of contamination such as untypical biofilm formation and/or clouding. Microbiological testing of plaque and waste fluid performed on Columbia blood agar (Sifin GmbH, Berlin, Germany) and Mitis salivarius bacitracin agar $(20 \%$ sucrose and 200 IU/1 bacitracin; BD Difco) revealed only the presence of streptococci.

Macroscopically, an opaque whitish surface was observed on the enamel surfaces in the control group not being protected by the varnish. Additionally, the control specimens

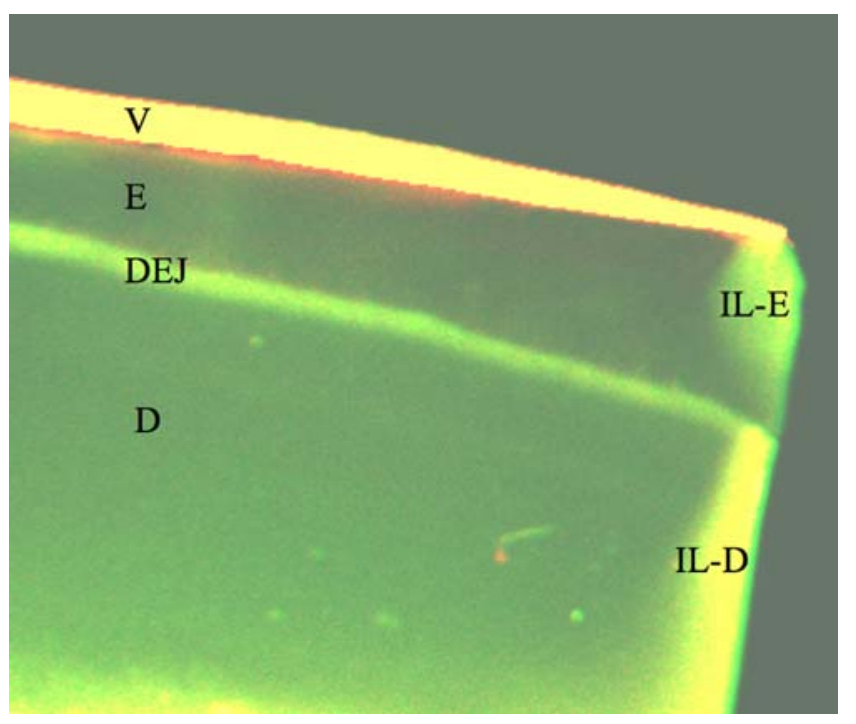

Fig. 2 Confocal laser scanning microscope image of a specimen from the test group 50 with varnish $(V)$ on the outer surface, an inner lesion in enamel $(I L-E)$, an inner lesion in dentine $(I L-D)$, enamel $(E)$, dentino-enamel junction $(D E J)$, dentine $(D)$ 
Table 1 Area of demineralization of the inner (wall) lesion in enamel and dentine

\begin{tabular}{|c|c|c|c|c|c|}
\hline Group & Gap width $(\mu \mathrm{m})$ & $\begin{array}{l}\text { Area of demineralisation in enamel } \\
(\text { mean } \pm \text { SD }) \times 1,000\left(\mu \mathrm{m}^{2}\right)\end{array}$ & $\begin{array}{l}\text { Statistical } \\
\text { significance }\end{array}$ & $\begin{array}{l}\text { Area of demineralisation in dentine } \\
(\text { mean } \pm \text { SD }) \times 1,000\left(\mu \mathrm{m}^{2}\right)\end{array}$ & $\begin{array}{l}\text { Statistical } \\
\text { significance }\end{array}$ \\
\hline Test group 250 & 250 & $90( \pm 24)$ & $p<0.05$ & $147( \pm 44)$ & n.s. \\
\hline Control group & 250 & $756( \pm 148)$ & & $168( \pm 48)$ & \\
\hline
\end{tabular}

n.s. not statistically significant $(p>0.05)$

showed visible loss of enamel tissue at the gap margin. Four specimens of the test groups completely lost their acidresistant varnish and three specimens partly lost their varnish. The loss of varnish resulted in the unintentional development of a primary caries lesion on one side of the specimen or the whole specimen, respectively. Test specimens with detectable primary lesions were excluded from the examination. Finally, 26 sites of test group 50, 23 sites of test group 100 and 27 sites of test group 250 were included in the study.

All specimens of the test groups with an intact varnish layer showed exclusively inner (wall) lesions in enamel and dentine (Fig. 2). These inner lesions spread into the dentine, undermining the enamel (Fig. 2). In the control group, both outer and inner lesions could be observed. The lesion area in enamel was significantly smaller in group TG 250 compared to group CG (Table 1). Blocking the development of an outer lesion had no influence on the extent of the inner lesion in dentin when using the selected initial gap width of $250 \mu \mathrm{m}$.

In the current in vitro setting, the gap width seemed to have a certain influence on the extent of the inner lesion when the development of an outer lesion is blocked. A statistically significant increase in lesion area could be detected in enamel between the gap width of 50 and $250 \mu \mathrm{m}$ and in dentine between 50 and $100 \mu \mathrm{m}$ as well as between 50 and $250 \mu \mathrm{m}$ (Table 1).

\section{Discussion}

The histopathological appearance of secondary caries with the presence of a characteristic lesion at the cavity wall (wall lesion) is explained by hydrogen ions of bacterial origin which diffuse into the microspace or which are directly produced by bacteria colonising the gap [3]. Both aspects deliver the rationale for the hypothesis that wider gaps allow the development of larger secondary wall lesions. However, the influence of the gap width is still a matter of debate, and conflicting results can be found in the literature [14, 16, 21-23]. Also, recent investigations in vivo and on extracted teeth were often criticised because the diagnosed caries in the tooth restoration interface was not supposed to be a secondary caries lesion but either an inner part of the outer lesion or a residual caries [12, 21, 24, 25]. Some investigators even doubt the existence of wall lesions as an own entity [11, 12].

In contrast to other studies on secondary caries in which the development of wall lesions was only investigated in combination with the development of outer lesions, the present study focussed on the isolated examination of wall lesions in relation to gap widths.

An independent development of outer and inner lesions has direct consequences on the clinical detection, treatment and prevention of secondary caries. If inner lesions do not form without the existence of outer lesions, diagnostic and preventive measures could concentrate on the outer lesion. If inner lesion form independently, "hidden caries" at the gap is possible and would need special concepts for its proper detection and management.

The results of this pilot study revealed that wall lesions in enamel and dentine could be produced in defined gaps between restoration and cavity wall of all specimens after an undisturbed acid attack produced by a plaque-like biofilm of $S$. mutans in an artificial mouth model. Inner lesions occurred even when the development of a primary lesion at the outer surface was inhibited. Thus, the inner lesion can be described as an own entity of caries lesion, independent from the outer lesion. The finding of Thomas et al. [10] in situ that "... secondary caries can occur in two indepen-

Table 2 Area of demineralisation of the inner (wall) lesion in enamel and dentine

\begin{tabular}{|c|c|c|c|c|c|}
\hline Group & Gap width $(\mu \mathrm{m})$ & $\begin{array}{l}\text { Area of demineralisation in enamel } \\
(\text { mean } \pm \text { SD }) \times 1,000\left(\mu \mathrm{m}^{2}\right)\end{array}$ & $\begin{array}{l}\text { Statistical } \\
\text { grouping }\end{array}$ & $\begin{array}{l}\text { Area of demineralisation in dentine } \\
(\text { mean } \pm \mathrm{SD}) \times 1,000\left(\mu \mathrm{m}^{2}\right)\end{array}$ & $\begin{array}{l}\text { Statistical } \\
\text { grouping }\end{array}$ \\
\hline Test group 50 & 50 & $73( \pm 13)$ & A & $117( \pm 32)$ & A \\
\hline Test group 100 & 100 & $78( \pm 14)$ & A, B & $142( \pm 36)$ & $\mathrm{B}$ \\
\hline Test group 250 & 250 & $90( \pm 24)$ & $\mathrm{B}$ & $147( \pm 44)$ & $\mathrm{B}$ \\
\hline
\end{tabular}

Groups with different letters mark a statistically significant difference $(p<0.05)$ 
dent locations: at the surface and along the wall" can be supported.

Due to several reasons, the results should be interpreted with caution: (1) a direct comparison between outer lesion inhibition and no outer lesion inhibition was performed just for a gap width of $250 \mu \mathrm{m}$, (2) it remains a matter of debate whether the inner lesion was produced by diffusion into the gap or by bacterial colonizing of the gap and (3) whether the results of this simplified bacterial-based model can be transferred to the in vivo situation. Own data generated with the present model show that gaps around $50 \mu \mathrm{m}$ and less are regularly colonised by S. mutans biofilms [26]. Further in situ studies should be carried out to focus on these questions.

Our data also revealed that the gap width between restorative material and cavity wall had a certain influence on the size of the wall lesion in an environment when the development of an outer lesion was inhibited (Table 2). Interestingly, a significant increase in wall lesion area could be observed in enamel only when the gap width was elevated from 50 to $250 \mu \mathrm{m}$. An increase from 50 to $100 \mu \mathrm{m}$ had no significant effect on the lesion size. In contrast to this, the wall lesion area in dentine ascended significantly with a gap enlargement from 50 to $100 \mu \mathrm{m}$, but did not increase significantly when the gap width was further elevated from 100 to $250 \mu \mathrm{m}$. One hypothetical explanation for this phenomenon might be that the dentine wall lesion in the present setting is more the result of the acids directly produced by the biofilm layer on top of the wall lesion in the gap, whereas the size of the wall lesion area in enamel is mainly driven by diffused acids produced by the undisturbed (no oral hygiene) and well-nourished biofilm outside the gap. This assumption is supported by our finding that specimens of the control group showed a drastically higher lesion formation than specimens from the test group 250, although the initial gap size was identical in both groups. Due to the caries attack by undisturbed biofilm development, an initial cavity developed at the enamel margins of the restoration in the control group, which was inhibited by the varnish in the test groups. This funnelshaped cavity gave elevated room for more biomass and, consequently, for a higher acid production with increased acid leakage. Since no oral hygiene measures were conducted to disturb the biofilm outside the gap and leaving the biofilm within the gap undisturbed, both sources of acid production cannot be considered independently. It seems obvious that wider gaps allow a faster bacterial colonisation of the initially sterile gaps in deeper areas, but it also seems obvious that beyond a certain threshold value (which would be around $100 \mu \mathrm{m}$ in the present setting), no further acceleration of colonisation speed can be expected. However, the gap widths of 50, 100 and $250 \mu \mathrm{m}$ where arbitrarily choosen to scan a range of clinically described gaps. Further studies would be needed including also gaps in the range of a few microns. Because possible threshold values most probably also depend on other factors such as saliva, type of biofilm, frequency of nutrients, extent of oral hygiene measures, the type of restorative material, etc., no direct extrapolation into the clinical situation is possible $[10,14,15,27]$.

Studies on effects of different gap widths on the extent of caries lesion have been conducted in vivo [13, 21], on extracted teeth [15], and in vitro with different bacterialbased caries models $[14,16]$. Each of these experimental designs has its advantages and disadvantages. Besides ethical considerations, in vivo and on extracted teeth, the individual caries risk of different patients cannot be controlled, it is difficult to distinguish residual from secondary caries, and it is difficult to determine and to standardise gaps. Even if the gap width can be measured on the outer surface, it must not continue in the same manner down to the bottom of the tooth restoration interface. Therefore, the use of bacterial-based in vitro caries model offers some advantages. Factors like a constant gap width, fluoridation, oral hygiene, ability of the dentist at making fillings, restoration materials and localisation of restoration can be standardised. However, we must be careful not to oversimplify the complex situation in secondary caries etiology just because of the supposed strong correlation between gap width and secondary caries $[15,25]$.

From the results of this investigation, we conclude that inner wall lesions of secondary caries can develop without the presence of primary (outer) lesions and therefore can be regarded as an entity on their own. In an environment in which the outer lesion development is inhibited, the extent of wall lesion increases with increasing gap width ranging from 50 to $250 \mu \mathrm{m}$.

Acknowledgements This study was supported by Marion von Zitzewitz and Steffie Balz who helped in the preparation of the specimens, Klaus Dannenberg from the "Medizinisch technische Labore" of the Charité by building the vice-like appliance, Dr. Carsten Grötzinger by helping us to take the CLSM images, Helmut Orawa from the "Institut für Biometrie und klinische Epidemiologie" of the Charité for his assistance in performing the statistical analysis. Special thanks to all of these people.

Conflict of interest statement The authors declare that they have no conflict of interest.

\section{References}

1. Baume LJ (1962) Special commission on oral and dental statistics: general principles concerning the international standardization of dental caries statistics. Int Dent J 12:65-75

2. Hals E, Andreassen B, Bie T (1974) Histopathology of natural caries around silver amalgam fillings. Caries Res 8:343-358 
3. Hals E, Nernaes A (1971) Histopathology of in vitro caries developing around silver amalgam fillings. Caries Res 5:58-77

4. Fontana M, Dunipace AJ, Gregory RL et al (1996) An in vitro microbial model for studying secondary caries formation. Caries Res 30:112-118

5. Garcia-Godoy F, Flaitz CM, Hicks MJ (1998) Secondary caries adjacent to amalgam restorations lined with a fluoridated dentin desensitizer. Am J Dent 11:254-258

6. Gilmour AS, Edmunds DH, Newcombe RG et al (1993) An in vitro study into the effect of a bacterial artificial caries system on the enamel adjacent to composite and amalgam restorations. Caries Res 27:169-175

7. Gilmour SM, Edmunds DH, Dummer PM (1990) The production of secondary caries-like lesions on cavity walls and the assessment of microleakage using an in vitro microbial caries system. J Oral Rehabil 17:573-578

8. Kidd EAM (1976) Microleakage in relation to amalgam and composite restorations. A laboratory study. Br Dent J 141:305310

9. Lobo MM, Goncalves RB, Ambrosano GMB et al (2005) Chemical or microbiological models of secondary caries development around different dental restorative materials. J Biomed Mater Res B Appl Biomater 74:725-731

10. Thomas RZ, Ruben JL, ten Bosch JJ et al (2007) Approximal secondary caries lesion progression, a 20 -week in situ study. Caries Res 41:399-405

11. Özer L (1997) The relationship between gap size, microbial accumulation and the structural features of natural caries in extracted teeth with class II amalgam restorations. Master thesis, University of Copenhagen, Denmark

12. Mjör IA, Toffenetti F (2000) Secondary caries: a literature review with case reports. Quintessence Int 31:165-179

13. Jorgensen KD, Wakumoto S (1968) Occlusal amalgam fillings: marginal defects and secondary caries. Odontol Tidskr 76:43-54

14. Derand T, Birkhed D, Edwardsson S (1991) Secondary caries related to various marginal gaps around amalgam restorations in vitro. Swed Dent J 15:133-138

15. Söderholm KJ, Antonson DE, Fischlweiger W (1998) Correlation between marginal discrepancies at the amalgam/tooth interface and recurrent caries. In: Anusavice K (ed) Quality evaluation of dental restorations. Criteria for placement and replacement, 1st edn. Quintessence Publishing, Berlin, pp 95-108

16. Totiam P, Gonzales-Gabezas C, Fontana MR et al (2007) A new in vitro model to study the relationship of gap size and secondary caries. Caries Res 41:467-473

17. Seemann R, Bizhang M, Klück I et al (2005) A novel in vitro microbial-based model for studying caries formation-development and initial testing. Caries Res 39:185-190

18. Seemann R, Klück I, Bizhang M et al (2005) Secondary carieslike lesions at fissure sealings with Xeno III and Delton - an in vitro study. J Dent 33:443-449

19. Shellis RP (1978) A synthetic saliva for cultural studies of dental plaque. Arch Oral Biol 23:485-489

20. Holm S (1979) A simple sequentially rejective multiple test procedure. Scand Statist 6:65-70

21. Hodges DJ, Mangum FI, Ward MT (1995) Relationship between gap width and recurrent dental caries beneath occlusal margins of amalgam restorations. Community Dent Oral Epidemiol 23:200204

22. Kidd EAM, O'Hara JW (1990) The caries status of occlusal amalgam restorations with marginal defects. J Dent Res 69:12751277

23. Rezwani-Kaminski T, Kamann W, Gaengler P (2002) Secondary caries susceptibility of teeth with long-term performing composite restorations. J Oral Rehabil 29:1131-1138

24. Kidd EAM, Fejerskov O (2004) What constitutes dental caries? Histopathology of carious enamel and dentin related to the action of cariogenic biofilms. J Dent Res 83(Spec No C):C35-C38

25. Özer L, Thylstrup A (1995) What is known about caries in relation to restorations as a reason for replacement? A review. Adv Dent Res 9:394-402

26. Seemann R (2005) Untersuchungen zur Kariespräventionin einem biofilmbasierten In-vitro-Modell (Habilitationsschrift). Universitätsklinikum Charité, Berlin, Germany

27. Goldberg J, Tanzer J, Munster E et al (1981) Cross-sectional clinical evaluation of recurrent enamel caries, restoration of marginal integrity, and oral hygiene status. J Am Dent Assoc 102:635-641 